\title{
Computer Aided Book Binding Design
}

\author{
Xia Zhi-Liang ${ }^{1}$, Tian Qi-Ming ${ }^{2}$ \\ Wenzhou Vocational \& Technical College, Wenzhou. 325035 \\ Xia441@sina.com
}

Keywords: materials;design;product design;innovative application.

\begin{abstract}
Books are the carrier of human civilization with the help of words, symbols and graphics to record the human thoughts and emotions, which telling the historical development process of human civilization. As an important part of books, book binding plays a very important role and has the independent aesthetic value. Because of that, bookbinding has formed its own spectacular design art.
\end{abstract}

\section{Parts of book binding design and its requirements}

The so-called book binding design can be understood as referring to the overall or the overall design. Its artistic refers to the overall design thought, not only about the painting design but also functional design. If the book biding designer only considers the painting, he must misunderstand why he designs and who he designs for.

Book binding design can be divided into two aspects of plane segmentation and 3-D segmentation. Plane segmentation means to study images, words and color composition in a picture. And 3-D segmentation referring to a book from outside to inside, each part has its functions and provides readers the fascinating experiences.

Cover design is the outward manifestation of the book-binding art through the form of art image design to reflect the contents of the books. Among wide variety of books on the bookshelves, the book cover plays a vital role as a silent salesman. Whether the cover is good or bad to a certain extent directly affects people's buying inclination. Hereby, graphics, colors and texts are the three elements of the cover design.

Head page: head page is very important, because it is close to the main body of the stories. So during the process of design, even a thread, a word, an abstract symbol, or one or two colors should be considered carefully. All elements should be meaningful and useful, not only with contents, but also with the sense of beauty.

End paper: the end paper is used to connect the book core and cover. No matter in the front or the back of a book, there is always a paper called end paper connecting cover and inside pages. It aims to keep the cover and inner pages sturdily connected and durable.

Main story body: the main story body is an important part of the book binding design.

Copyright page: the copyright page usually is printed at the opposite of the head page or the opposite of the last blank page. The words mostly locate at the bottom of the copyright page.

\section{The book sizes}

At present there are four kinds of commonly used paper specifications: including $787 * 1092 \mathrm{~mm}$. $850 * 1168 \mathrm{~mm}, 880 * 1230 \mathrm{~mm}$ and $889 * 1194 \mathrm{~mm}$. A full size paper is cut into multiple equal width sheets and the quantity of sheets is the number of paper. For example, a piece of full size paper is cut into equal width of 16 pieces of paper, called number 16 . If the full size paper is cut into 32 pieces of paper, it is called number 32 and so on. Due to a variety of paper sizes, the same number paper may not be the same size. For example, the copyright of a book prints " $787 * 10921$ / 16 ", which means a full paper size of $787 * 1092 \mathrm{~mm}$ been cut into 16 pieces. If the copyright of a book prints" $850 * 11681 / 16$ ", which means a full paper size of $850^{*} 1168 \mathrm{~mm}$ been cut into 16 pieces. In 
order to distinct paper with the same number but not the same size, usually called " $787 * 10921 /$ 16 "size 16 , and called "850*1168 1/16" lager 16.

Recently the most commonly used book sizes as shown in table 1.

Table 1. Recently the most commonly used book sizes

\begin{tabular}{|c|c|c|c|}
\hline 8 & 260 & 376 & $787 \times 1092$ \\
\hline BIG 8 & 280 & 406 & $850 \times 1168$ \\
\hline BIG 8 & 296 & 420 & $880 \times 1230$ \\
\hline BIG 8 & 285 & 420 & $889 \times 1194$ \\
\hline 16 & 185 & 260 & $787 \times 1092$ \\
\hline BIG16 & 203 & 280 & $850 \times 1168$ \\
\hline BIG16 & 210 & 296 & $880 \times 1230$ \\
\hline BIG16 & 210 & 285 & $889 \times 1194$ \\
\hline 32 & 130 & 184 & $787 \times 1092$ \\
\hline BIG 32 & 140 & 203 & $850 \times 1168$ \\
\hline BIG 32 & 148 & 210 & $880 \times 1230$ \\
\hline BIG 32 & 142 & 210 & $889 \times 1194$ \\
\hline 64 & 92 & 126 & $787 \times 1092$ \\
\hline BIG 64 & 101 & 137 & $850 \times 1168$ \\
\hline BIG 64 & 105 & 144 & $880 \times 1230$ \\
\hline BIG 64 & 105 & 138 & $889 \times 1194$ \\
\hline
\end{tabular}

\section{The book binding}

Binding is a whole process from collecting pages to sealing, including sorting the printed pages according to the order, connection, stitching, putting on covers etc.

3.1 Saddle stitching: It is also called saddle iron wire stitching, putting the covers and pages all together. See picture1.

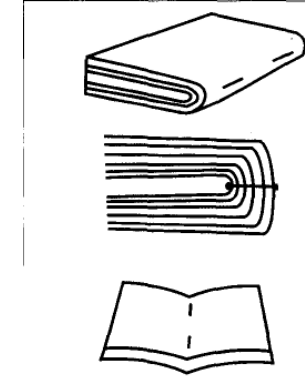

Picture1. Saddle stitching

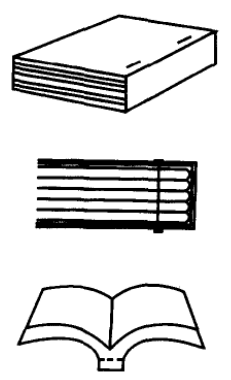

Picture2. Flat stitching

3.2 Flat stitching: presses the orderly folded pages tightly together and stitches them with sewing thread or nail wire from the front of the book to the bottom, forms a core, and then add the covers, finally cut book. See in picture 2 .

\subsection{Thread stitching}

By principle of thread crossing connection at the creasing place of book spine, each packed pages are locked with each other.

\subsection{Loose page stitching}

Punching holes at the stitching place of a book, and then with spring metal coil or threaded coil locked the holes together.

Hardcover is a kind of book more delicate and complicated. The materials used for hardcover books are more elegant than the ones for the paperback. The book binding of hardcover is stronger and more durable. The hardcover is especially suitable for the books with high quality requirements, more pages, repeated reading requirements, and with the saving value for long period of time. There are two kinds of hardcover books, including hard book binding and soft book binding. The covers of 
hardcover books can use different materials, printing methods, different styles and effects. There are many materials can be used for covers, such as all kinds of fabrics, leathers etc in addition to the paper.

\subsubsection{The layout of book-binding}

Layout of book-binding means the arrangement and design for the fonts and diagrams on each page. A page often is left with blank space around four corners, and the words and diagrams locate in the middle, which is called print area. Upper blank part of the print area is named sky (sky head), lower blank part of the print area is named earth (earth foot), and the areas close to the cutting and stitching are respectively called cutting mouth and stitching mouth. See picture3.

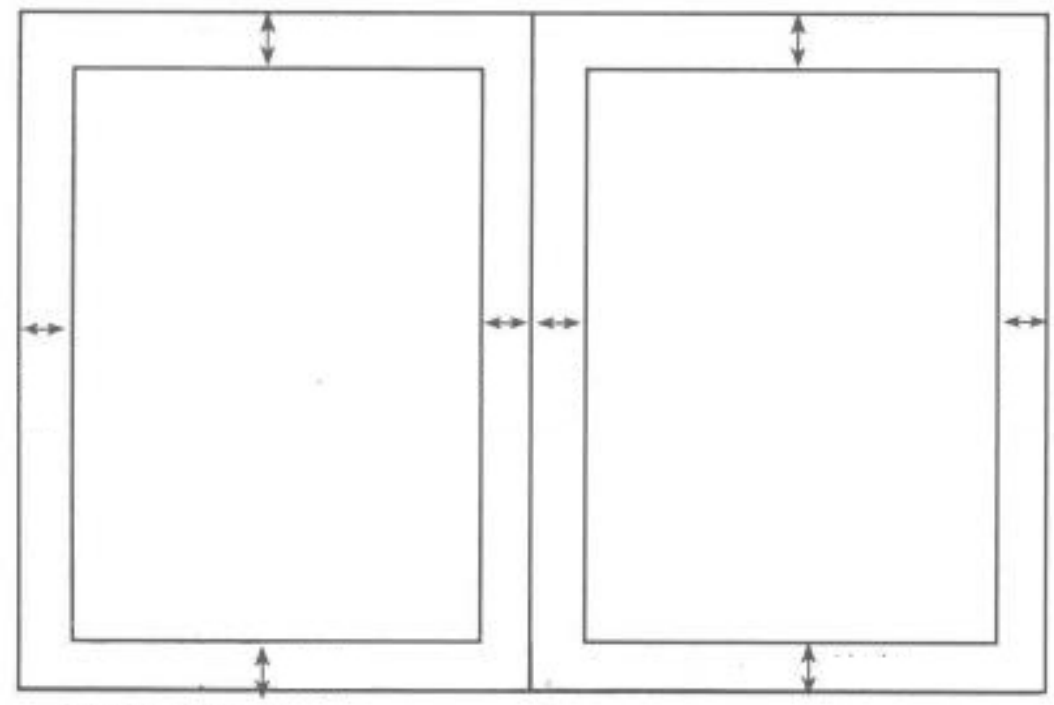

Picture3. The book layout

\subsubsection{The book binding principle}

Book binding design is a whole process from the manuscripts to books being published, is also from the form of the plane to the form of three-dimensional. It contains a series of system designs such as the artistic thinking, design ideas and techniques. It also contains the sizes of the books, forms of binding, covers, fonts, layout, colors, illustrations, paper materials, printing, binding and process of each art design.

\subsubsection{Add Chinese factors into the color design}

The information age makes the design art continuously interconnected with many other elements, just like interconnected branches of rivers moving forward and without stopping. Constantly emerging new materials and new technologies make our eyes too busy to see all.

Although designers are experienced and good at in mastering all design elements, however, affected by the old mindset, the designer's ideas are always confined into contemporary visual elements as the key of design, and ignored design art enlightenment from traditional elements.

\subsubsection{Characteristics and the use of traditional colors}

With the help of teacher's instruction and my understanding of the topic, considering that the native colors are most comfortable and acceptable to users, I preliminarily plan to use the traditional colors. According to the five staple elemental colors of nature, I use the color of red, white, black, green and yellow to determine the colors of the book binding design, which can arouse users' enthusiasm. See picture4. 

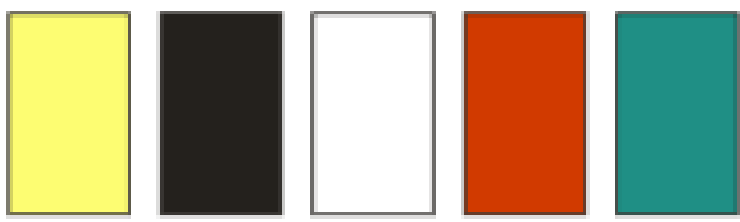

Picture4.five staple elemental colors

\subsubsection{Composition form of Chinese factors}

"Freehand brushwork" means unnecessary for detailed description, but focuses on the performance of the artistic conception. My layout version design is to organically combine the colors, words, and pictures together in order to match with the topic. Even though freehand of traditional China does not contain much detailed over labor, the purposefully arrangements are unique, see picture5, 6 .
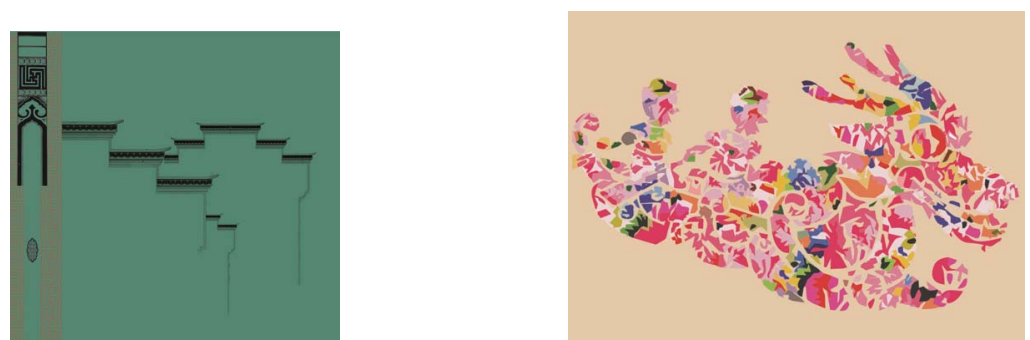

Picture5. The arrangements of colors and paintings Picture6. Elements arrangements

The layout should apply to the principles of balance and symmetry, contrast and point of view.

Even though with different concepts, balance and symmetry are the basis for the composition. Both of them can make art with stability. Stability is visual habits and aesthetic concept, which is formed by the long-term observation of nature. Balance and symmetry representing logical proportional relationships cannot be equated with the average.

Average is stable, but lacks the aesthetic feeling of change. Symmetry is with strong sense of stability, but there are fewer changes than balance. Although symmetry is an important principle for composition, it is unpractical.

The advantage of contrast is not only to enhance the artistic appeal, but also reflect and sublimate the theme.

The point of view is the term from perspective science, also named vanish point. The point of view draws people's attention into the same point which can interpret the theme. No matter where it put, the extension lines from all the objects should point to this point.

We got the types of composition through the three principles. 1)Horizontal type, which makes the image stable and strong. 2)Vertical type, which makes the image serious and modest. 3) S-type, which makes image elegant and with changes. 4)Circle type, which makes image saturated and with tension. 5)Radiation type, which makes the image with the sense of depth. 6)Center type, which makes theme clear with strong effect. 7)Gradual type, which makes the image with a sense of rhythm. 8)Scattered dots type, which makes image with a sense of freedom.

\section{Conclusion}

Visual language as a carrier of the artistic thought is developed and innovated continuously in the history. Chinese nation has cultural inheritance of five thousand years, and the history left us a lot of cultural elements. Each traditional element contains a profound cultural accumulation, engraved by a deep national mark. They are the fruits of the wisdom of Chinese people----great and profound. Some people say that "what belongs to the nation is what belongs to the world." Although this point is still argued until now, but undoubtedly, every country's exploring and protecting their own national culture has reached an unprecedented state. Under today's zero border cultural environments, "fast 
food culture" and "cultural infiltration" penetrates to the every corner of the world. By contrast, the values of the distinctive traditional cultures of each country look more important day by day.

\section{References}

[1] Jiang Zhongzhi, The Artistic and Commercial Features of Book Cover Design [J]. Chongqing: Packaging Engineering, October, 2010

[2] Deng Zhonghe; Creative Design of Book Binding and Layout [M]. China Youth Press, January, 2004.

[3] Wang Kaiqing, The Book Binding and Layout of Contemporary China [J]. Chongqing: Packaging Engineering, April, 2006.

[4] G. Henkelman, G.Johannesson and H. Jónsson, in: Theoretical Methods in Condencsed Phase Chemistry, edited by S.D. Schwartz, volume 5 of Progress in Theoretical Chemistry and Physics, chapter, 10, Kluwer Academic Publishers (2000).

[5] Wang Mei. On the Design of Book Spine [J]. Beijing: China Publishing Journal, September, 2010 . 